\title{
3D Odor Source Localization using a Micro Aerial Vehicle: System Design and Performance Evaluation
}

\author{
Chiara Ercolani ${ }^{1}$ and Alcherio Martinoli ${ }^{1}$
}

\begin{abstract}
Finding chemical compounds in the air has applications when situations such as gas leaks, environmental emergencies and toxic chemical dispersion occur. Enabling robots to undertake this task would provide a powerful tool to prevent dangerous situations and assist humans when emergencies arise. While the dispersion of chemical compounds in the air is intrinsically a three-dimensional (3D) phenomenon, the scientific community tackled primarily two-dimensional (2D) scenarios so far. This is mainly due to the challenges of developing a platform able to successfully provide chemical compounds samples of a 3D space. In this paper, a 3D bioinspired algorithm for odor source localization, previously validated in a controlled physical environment leveraging a robotic manipulator, is adapted for deployment on a micro aerial vehicle equipped with an odor sensor. Given the effect that the propellers have on a gas distribution, the algorithmic adaptation focused on enhancing the sensing strategy of the platform. Additionally, two sensor placement configurations are assessed to determine which one yields best sensing results. A performance evaluation in different environmental scenarios is carried out to test the robustness of the implementation. Two different localization systems are used for the performance evaluation experiments to quantify the impact of localization accuracy on the algorithm outcome.
\end{abstract}

\section{INTRODUCTION}

When gas leaks, toxic chemical dispersion or environmental emergencies occur, Odor Source Localization (OSL) could play a crucial role in taking fast and effective countermeasures. The scientific advances in the fields of robotics, embedded systems and chemical sensing allow OSL to be performed by robots and sensor nodes equipped with odor sensors. The vast majority of the research in robotic OSL so far employs ground robots capable of navigating on a $2 \mathrm{D}$ plane [1]. This is mainly due to the fact that platforms capable of 3D motion usually displace using propellers, which impact greatly the gas distribution and hinder the sensing abilities of the system. However, since odor dispersion is inherently a 3D phenomenon, effectively extending odor source localization to the third dimension is an important direction for this field of research.

In recent years, Unmanned Aerial Vehicles (UAVs) have been equipped with gas sensing capabilities and used for odor source localization and mapping tasks [2], [3]. The main challenge when using UAVs for OSL is that the turbulence created by the drone's propellers, called "wake", has an

\footnotetext{
*This work was funded by the Swiss National Science Foundation under grant 200020_175809/.

${ }^{1}$ The authors are with the Distributed Intelligent Systems and Algorithms Laboratory, School of Architecture, Civil and Environmental Engineering, École Polytechnique Fédérale de Lausanne (EPFL), 1015 Lausanne, Switzerland
}

effect on the plume of the gas, making its detection a challenging task [4], [5], [6].In order to avoid the effect of the wake, Neumann et al. [7] proposed a robotic platform for aerial sensing that uses remote gas sensors. However, the start-stop strategy for gas detection proposed in this paper does not allow continuous detection and drains the battery quickly. Moreover, quadrotors of large dimensions are not suited for deployment in indoor and cluttered environments. In [8], sensing was achieved by having a human operator halt the propellers when the drone is close to the source to enhance odor detection. However, this operation mode, called "butterfly", does not scale well to large scenarios and does not fully exploit the capabilities of the drone. Other flying vehicles, such as blimps, whose propellers are less impactful on the plume since they are not used to maintain buoyancy, are used for gas localization and mapping [9]. Although blimps allow for a longer flight time, these vehicles do not present the same range of maneuverability and versatility as rotary-wing aerial vehicles.

Micro Aerial Vehicles (MAVs) offer an interesting alternative to traditionally bigger drones, while maintaining similar flight dynamics properties. Given the smaller size of the propellers, their impact on the plume does not hinder odor detection as much as with UAVs and makes them a viable alternative for odor source localization [5], [10]. Moreover, they are well-suited for deployment in indoor and cluttered environments, such as factories or chemical plants. Shigaki et al. [5] achieve satisfactory sensing results by placing sensors on the sides of two drone propellers and emulating the sensing strategy of the silkworm moth. In [10] the authors were able to extract an odor map that indicates the position of the source from the data by gathering data while the MAV flies on a predefined path at fixed heights. Although no algorithm was implemented to define the path of the MAV, their work demonstrates that a rough approximation of the location of the odor source can be obtained in a short time. Thus, micro drones, even with their limited flight time capabilities, can be good candidates for odor source localization and mapping.

Interesting considerations can be made about the optimal sensor placement to improve the sensing capabilities of a UAV. In [11], Neumann et al. use the airflow of the propellers to convey odor particles to the sensors placed below them. This solution was identified as the best trade-off between a long carbon fiber tube that would avoid the turbulence entirely and placing the sensor directly at the bottom of the drone. This conclusion was also reached in [8], where a micro drone is equipped with a gas sensor and a qualitative 
study is conducted to identify an optimal position for the sensor placement. However, this solution affects the stability of the drone, especially when applied to smaller vehicles. Villa et al. [6] tried to evaluate the air flow to determine where a gas sensor should be mounted on a drone. Their findings suggest that the sensor should be placed as far away from the center of the UAV as possible to avoid decreasing the sensor's capabilities. This is not always possible, thus a trade-off between optimal placement and feasibility is recommended. In [5], particle image velocimetry is used to visualize the airflow around the quadcopter. The aim of this analysis is to validate the chosen configuration of sensors (two on the front side of the quadrotor) that was selected for this work. Unfortunately, one cannot draw conclusions about the optimal placement in general. One interesting consideration is that the authors suggest that placing a chemical sensor where the wind speed is highest might not be beneficial, since the sensor's heater cools down and does not react to odor as well. A consensus is not reached as of what the best sensor placement is, while also taking into consideration the feasibility of the implementation. In this work, two sensor placements are evaluated: on top and at the bottom of the drone's body. These were chosen because they are the most straightforward to implement and the results obtained in [10] with a similar setup suggested that a more elaborate sensor placement on a MAV might not be necessary.

Odor source localization algorithms often take inspiration from natural phenomena [12]. In fact, many living species such as moths, bacteria and dung beetles rely on the ability of localizing an odor source for survival [13], making bioinspired algorithms optimized for the end goal. Bio-inspired algorithms require low computational resources and a small memory, and are very reactive. This makes them good candidates for deployment on UAVs in general and on MAVs in particular, as they usually present limited resources in terms of flying time and computational capabilities. Neumann et al. [11] compares three bio-inspired algorithms for odor source localization implemented on a UAV: surge-cast [14], zigzag [15] and pseudo-gradient-based [16]. All three algorithms were successful in real world experiments, with zig-zag and pseudo-gradient-based performing slightly better. However, this evaluation was carried out with a larger drone $(1 \mathrm{~m}$ diameter), whose motion was limited to a $2 \mathrm{D}$ plane in the air, and without exploiting the full range of motion available when using an aerial vehicle. Thus, a conclusion on which type of bio-inspired algorithm would perform better in a 3D environment cannot be drawn. An experimental evaluation of a three dimensional algorithm with a MAV was presented by Shigaki et al. [5]. The algorithm proposed is inspired by the behavior of the silkworm moths [17]. The experiments assume that the quadrotor starts two meters away from the source and in front of it, at different heights. Better success rate were obtained when the drone's initial position was lower than that of the source due to the fact that ethanol, used for these experiments, is heavier than air. The biggest limitation of this approach is that the chances of failure increase when the drone does not detect chemical compounds fast. Moreover, the fixed starting position in front of the plume and the limited testing area provide a highly simplified environment.

Rahbar et al. [18] proposed a 3D bio-inspired algorithm that uses a Lévy Walk algorithm to initially find the plume and then proceeds to follow the plume with a upwind surge. If the plume is lost, a 3D spiraling motion, inspired by moths, is used to reacquire the odor [14], [19]. The algorithm was tested in a wind tunnel, which provides controllable quasilaminar flow, and a comparison between the surge-cast and spiral method in 2D and in 3D highlights the fact that a three dimensional approach yields a robust localization of the source. However, the validation, which was carried out with a small-scale wheeled robot mounted on a traversing system used to emulate a $3 \mathrm{D}$ motion, does not tackle the challenges of a deployment on a platform that could be exploited in realworld scenarios, such as localization, sensor integration and in particular some algorithmic adaptations to account for the effect of the wake of the propellers on the gas distribution.

The contribution of this work is therefore the adaptation of the 3D bio-inspired algorithm reported in [18] for deployment on a MAV and an evaluation of its performance in a controlled environment offering a good compromise between repeatability and realism. Moreover, this paper will compare the results of odor source localization with two different sensor placements shown in Figure 1. A performance evaluation in different environmental conditions is also carried out, together with an evaluation of the impact of two different localization systems on the performance of the algorithm. No previous work presents an in-depth evaluation of a 3D bio-inspired algorithm on a MAV equipped with sensing capabilities. Additionally, the deployment is carried out in a sizable area compared to the drone's size, is not limited to a 2D plane, and exploits the whole range of motion of the MAV, making this contribution an important step towards the application of 3D OSL in real-world scenarios. The following sections of the paper outline the proposed method, describe the experimental setup and present the results obtained.

\section{MATERIALS AND METHODS}

This section describes the platform used for this work, a MAV equipped with sensing capabilities, and presents the algorithm implemented. Moreover, it introduces the localization strategies used throughout the performance evaluation.

\section{A. Micro drone}

To accomplish the task of 3D odor source localization, a commercially-available MAV was equipped with sensing capabilities (Figure 1). A Crazyflie V2.1 (CF2, Bitcraze AB, Malmö, Sweden) was chosen due to its small dimensions $(10 \times 10 \mathrm{~cm})$, open hardware and software architecture and because this drone has already been successfully employed for odor localization and mapping tasks in literature [4], [10]. The CF2 is built with development in mind and provides access to power lines, $\mu \mathrm{C}$ pins and communication buses. The main limitations of this platform are the flight time of up to 7 minutes and a maximum payload of $15 \mathrm{~g}$. 


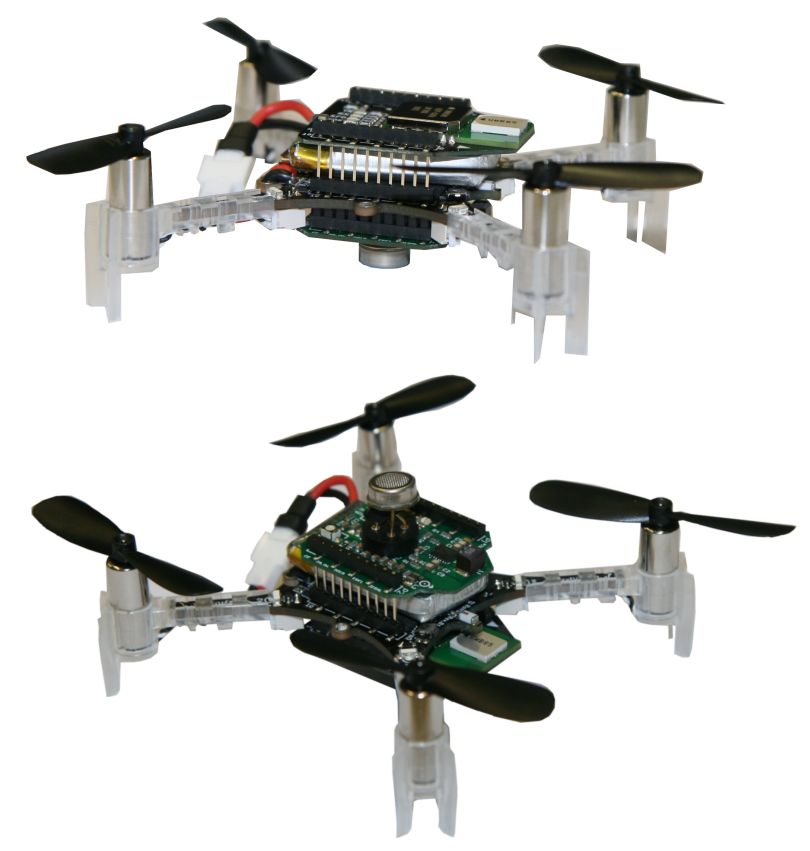

Fig. 1. Crazyflie V2.1 equipped with a custom PCB for odor sensing. Both sensor configuration (top and bottom) are presented.

A custom Printed Circuit Board (PCB) was developed to provide sensing capabilities to the $\mathrm{CF} 2$. The PCB hosts a MICS-5521 CO/VOC sensor [20] and an STM8L151F3U6TR ultralow power micro-controller used to convert analog data coming from the sensor to digital with a sampling frequency of $10 \mathrm{~Hz}$. The $\mathrm{I}^{2} \mathrm{C}$ bus is used to send odor data coming from the sensor to the CF2 microcontroller.

\section{B. Algorithm}

The algorithm selected for this implementation is the one described in [18]. The algorithm is composed of three parts: plume finding, plume following and plume acquisition. During plume finding, a Lévy Walk is performed on a crosswind plane to acquire the plume. In this work, the wind is assumed quasi-laminar and parallel to the $\mathrm{Y}$ direction, making the $\mathrm{X}-\mathrm{Z}$ plane the crosswind one. When the plume is found (i.e. the odor concentration is above a given threshold), an upwind surge is performed in the $\mathrm{Y}$ direction to follow the plume. When the plume is lost (i.e. the odor concentration is below the same threshold), a 3D spiraling motion on the $\mathrm{X}-\mathrm{Z}$ plane is used to reacquire it. The source is considered reached when the robot is in the plume and reaches an area of $40 \times 40 \times 30 \mathrm{~cm}^{3}$ around the source.

While the general structure of the algorithm is the same as in [18], some adaptations were necessary to enhance the sensing strategy of the MAV and compensate for the propeller effect. In [18], the robot stops at the end of each step to sample odor values in order to reduce noise during data acquisition. However, in this work, the drone does not stop to sample because of the great impact that hovering has on the plume. As a result, sampling is done continuously while in motion. Additionally, given the lower quality of the acquired data, the maximum odor value within a sliding window is used to decide on the next step of the algorithm, rather than an average of the samples. Although averaging is an effective method to improve sensing in a noisy setting, in this scenario it is more important to avoid missing a cue about the presence of the plume than to low-pass filter the signal. The spiral drift constant, which defines the distance between the laps of the spiral movement, was also lowered. In fact, the plume can be lost due to the propeller effect and a denser spiral allows to linger for a longer time closer to where the plume was last sensed. Given the tight time constraints imposed by the flight time of the $\mathrm{CF} 2$, an initial scanning to determine the odor threshold that identifies whether the plume is detected or not is not possible. For this reason, the threshold is defined a priori. The velocity of the CF2 is kept constant at $0.3 \mathrm{~m} / \mathrm{s}$ and the size of the step that the MAV can take at each iteration of the algorithm is $0.1 \mathrm{~m}$. This step size was chosen to limit the oscillations of the drone.

\section{Implementation details}

Two localization strategies are used for the experimental evaluation. The first one exploits an Ultra Wide Band (UWB) system called Loco Positioning System, also built by Bitcraze. The system is based on the Decawave DWM1000 chip and has a reported accuracy in the $10 \mathrm{~cm}$ range. To provide coverage to the desired area, 8 UWB anchors were deployed and used for localization. An UWB tag is placed on the CF2 to allow the quadcopter to acquire its position. An Extended Kalman Filter, exploiting UWB and Inertial Measuring Unit (IMU) data, is used to improve the estimation of the position. The second localization strategy exploits a Motion Capture System (MCS) from Motion Analysis ${ }^{1}$ consisting of 13 Kestrel 1300 cameras deployed inside the wind tunnel. Five passive markers of diameter of $10 \mathrm{~mm}$ are placed on the CF2 to allow localization. MCSs offer very precise localization measurements, with accuracy of around $1 \mathrm{~mm}$. However, they are significantly more expensive than an UWB system such as the one used during this project. Moreover, an UWB system can be deployed more quickly even in realistic environments (e.g. outside a laboratory or outdoors), and represents up to date the best localization system in non line of sight conditions, due to the capabilities of a large spectrum of electromagnetic waves to penetrate a large palette of materials. The objective of the comparison between the two localization systems is that of quantifying the effect that a more precise localization has on the algorithm performance and whether an UWB solution can provide satisfactory results.

The CF2 communicates with the ground station (a PC equipped with a USB antenna) over the $2.4 \mathrm{GHz}$ ISM radio band. When using the UWB localization system, the CF2 sends position and odor data to the ground station and receives a command to move to the next waypoint based on

\footnotetext{
${ }^{1}$ https://www.motionanalysis.com/
} 
the algorithm described previously. When the MCS is used, the position data is acquired by the MCS software and sent to the CF2 through the radio link.

The payload of the CF2 includes the custom PCB board and the UWB tag board or MCS markers. The total weight of the payload is $6.5 \mathrm{~g}$ in both cases. Observations during preliminary experiments estimated a flight time of 4 to 5 minutes in these conditions, depending on the maneuvers performed by the vehicle.

\section{EXPERIMENTAL EVALUATION}

Two sets of experiments were carried out to evaluate the performance of the MAV. The first set of experiments tries to determine the optimal sensor placement between the top and the bottom of the body of the quadcopter. The second sets of experiments evaluates the performance of the algorithm in different conditions, and using the best sensor placement determined previously. The first set of experiments was carried out using the UWB localization system, while the second set was repeated also with the MCS system.

\section{A. Sensor placement}

The versatility of the CF2 platform allowed to place the custom PCB above or below the drone with only a minor change. The two configurations can be seen in Figure 1. Ten experiments were carried out with each configuration and the results were compared. The aim was to decide which sensor placement works best among the two that are easier and more immediate to implement. To create favorable environmental conditions, the wind speed during these experiments is set to high $(0.7 \mathrm{~m} / \mathrm{s})$, since the work in [18] presented evidence that the algorithm is sensitive to low wind speed. While the source release rate was found to be less significant on the impact of the algorithm in [18], we chose the high release setting for this set of experiments.

\section{B. Performance evaluation}

After selecting the best sensor placement, a performance evaluation was carried out to verify the robustness of the algorithm implemented on the MAV in different environmental conditions. This section describes the parameters chosen and their expected impact on the experiments as documented in [18]. Five experiments were carried out for each setup. The parameters of the setups tested and their values are presented in Table I.

TABLE I

SETUP PARAMETERS USED IN THE PERFORMANCE EVALUATION

\begin{tabular}{|c|c|c|}
\hline Setup & Wind Speed & Release Rate \\
\hline A & $0.7 \mathrm{~m} / \mathrm{s}$ & high \\
\hline B & $0.2 \mathrm{~m} / \mathrm{s}$ & high \\
\hline C & $0.7 \mathrm{~m} / \mathrm{s}$ & low \\
\hline D & $0.2 \mathrm{~m} / \mathrm{s}$ & low \\
\hline
\end{tabular}

1) Wind speed: The strength of the wind impacts the width of the plume. For stronger wind, the plume is narrower, making the acquisition of the plume more challenging. However, once the plume is found, the task of following it is supposedly easier, since the robot is well positioned to find the source. Theoretically, this does not happen with lower wind and a wider plume. In fact, in this scenario, the robot might acquire the plume faster, but lose it several times before reaching the source. The conclusions in [18] support this claim.

In this paper, the effect of the wind speed when using a MAV is studied. The wind speed used are 0.2 and $0.7 \mathrm{~m} / \mathrm{s}$. The higher wind speed setting is lower than the one used in [18], which was $0.9 \mathrm{~m} / \mathrm{s}$, because the CF2's flying capability is impacted greatly by the wind conditions. These values are coherent with real world scenarios.

2) Source release rate: The source release rate affects how dense the plume is. Theoretically, a higher source release rate produces a denser plume, which makes the detection easier for the robot. However, in [18], this parameter was found experimentally to be less critical than wind speed.

The gas used for the experiments is ethanol and it is released with a stationary electric pumping device. Two values for source release rate are chosen for this work: 0.5 $\mathrm{L} / \mathrm{min}$ (high) and $0.25 \mathrm{~L} / \mathrm{min}$ (low). These values correspond to the mix of air and evaporated ethanol that is released by the pump. The ratio of ethanol to air is not known. The traces of ethanol released in the air are not detectable by a human nose. The values chosen for this set of experiments are higher than the ones used in [18], which were 0.1 and $0.2 \mathrm{~L} / \mathrm{min}$ respectively, to facilitate the sensing for the MAV.

\section{Evaluation Metrics}

Two metrics are used to quantify the performance of the algorithm in the different scenarios: success rate and distance overhead.

A successful run occurs when the MAV can find the source of the odor within 4 minutes and 40 seconds from the start of the experiment. This timeout value was chosen based on the flight time capabilities of the drone with a payload of 6.5 g. The success rate $s_{r}$ is defined as the number of successful runs over the total number of runs:

$$
s_{r}=\frac{\# \text { successful runs }}{\# \text { runs }}
$$

To understand how well the algorithm is performing compared to the best case scenario in which the robot goes straight to the source, the distance overhead $d_{o}$ is used. This metric was introduced in [21] and is computed by the distance effectively travelled by the robot, $d_{t}$, divided by the shortest upwind distance, $d_{u}$ :

$$
d_{o}=\frac{d_{t}}{d_{u}}
$$

In this setup, the upwind distance is $10.72 \mathrm{~m}$ and is defined as the distance between the source and the optimal starting point of the robot, in front of the source at minimum height. 
As the velocity of the drone is kept constant $(0.3 \mathrm{~m} / \mathrm{s})$, the travelled distance information allows for computing the time taken to reach the source.

\section{Experimental setup}

1) Wind tunnel setup: The experiments were carried out inside a wind tunnel of volume $4 \times 18 \times 2 \mathrm{~m}^{3}$. The wind flow inside the wind tunnel can be adjusted as desired and laminarized by a dedicated honeycomb filter. The experiments were carried out with a wind flow of $0.2 \mathrm{~m} / \mathrm{s}$ and $0.7 \mathrm{~m} / \mathrm{s}$, in a quasi-laminar regime. The volume of the tunnel effectively used for the experiments is $2.5 \times 10.8 \times 0.8 \mathrm{~m}^{3}$. Limitations in volume are due to the fact that the UWB system presents several blind spots closer to the anchors. Moreover, the wind tunnel is an almost entirely metallic structure, which affects the accuracy of the UWB and contributes to limiting the usable area. The same experimental volume was used for experiments with the MCS, although this system offers better coverage inside the tunnel. The source is placed at a height of $0.82 \mathrm{~m}$.

2) Starting position: In all the experiments that were carried out, the drone is placed on the ground in the same corner of the volume, far from the source. The $\mathrm{x}$ coordinate of the starting position is randomized for every experiment, while for $\mathrm{z}$ and $\mathrm{y}$ the minimum value is chosen. The minimum y value is chosen so that the robot performs the Lévy Walk on the same crosswind plane each time, while the same $\mathrm{z}$ was chosen as a simplification and to save time overhead at the beginning of the experiment.

\section{RESUlTS}

This section outlines the results obtained when comparing two sensor placement positions and presents an in-depth performance evaluation of the system in different environmental conditions and using two localization strategies.

\section{A. Sensor Placement Results}

The first set of experiments was meant to determine where to place the sensor on the MAV. Figure 2 shows that the success rate is higher when the sensor is placed at the bottom of the CF2. Moreover, Figure 2 shows that the distance overhead is smaller with the bottom configuration, and presents a lower variability. From these results it seems that the down-washing effect of the propellers is conveying odor particles towards the bottom sensor. This conclusion is in line with the results presented in [11] and [8]. No tube is used to place the sensor further away from the drone, a solution that increases vehicle stability and eases the integration of the sensor on the MAV.

\section{B. Performance Evaluation Results}

The second set of experiments evaluated the robustness of the algorithm to different wind speeds and source release rates. The results obtained with the UWB localization system will be presented first. All runs in each setup gave a successful outcome. Figure 3 shows the distance overhead for the four setups. Setup B and D, the ones with lower wind speed,
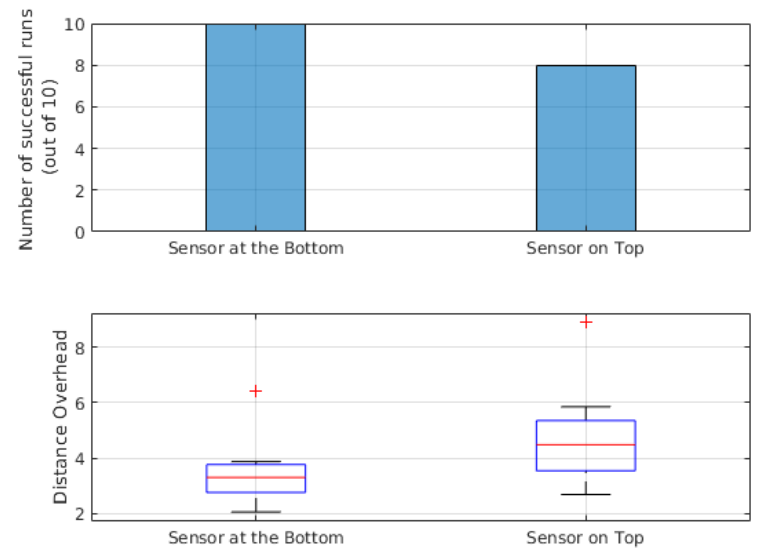

Fig. 2. Top picture: success rate for the sensor placement at the bottom and on top of the MAV. Bottom picture: distance overhead for the sensor placement at the bottom and on top of the MAV.

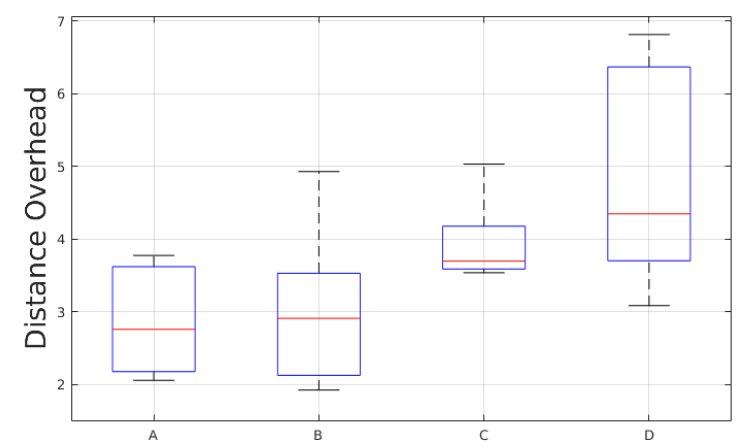

Fig. 3. Distance overhead for the performance evaluation experiments using a UWB localization system and in different setups as presented in Table I.

present a higher variability of data. Moreover, experiments $\mathrm{C}$ and $\mathrm{D}$, where the source release rate is low, have a higher mean distance overhead. In general, all configurations yield good results, with outstanding performance in case of high release rate and high wind speed. On average, the time taken by the MAV to reach a source located $10.72 \mathrm{~m}$ away is about two minutes. The results in [18] indicate that the algorithm seemed to be negatively affected the most by low wind speed, while in this scenario it seems that a low release rate has a greater negative impact. This could be explained by the considerable sensing difficulties faced by the MAV, which are enhanced by the lower presence of particles in the air. Slight changes in the setup, such as higher release rate and different wind speeds, that were made to improve the sensing strategy of the CF2, do not allow a direct quantitative comparison between the results presented in this paper and those in [18]. The same odor threshold was used for all the experiments. In the future, it would be interesting to explore what the impact of a lower odor threshold is with lower wind speed and low source release rate.

The biggest limitation of this setup is the accuracy of the 


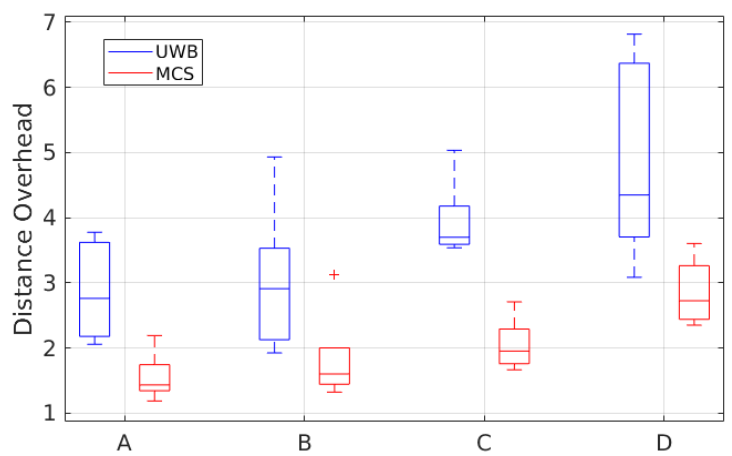

Fig. 4. Distance overhead for the performance evaluation experiments with an UWB and MCS localization strategy in different setups as presented in Table I (UWB in blue and MCS in red).

UWB-based localization system on which the MAV mainly relies on for navigation. In particular, accuracy seemed to drop closer to the ground and the oscillations on the Z-axis were sometimes affecting the performance of the algorithm. For this reason, the second set of experiments was repeated using a MCS for localization. All runs gave a successful outcome, except for one with setup $\mathrm{D}$, in which the robot timed out before reaching the source. This can be explained by the fact that this setup is the hardest one for sensing and it was challenging for the MAV to reacquire the plume. Figure 4 shows a comparison between the two localization systems in the different scenarios presented in Table I. It can be observed that the results from the two localization systems seem to follow similar trends, with performance degradation in case of scenarios $\mathrm{C}$ and $\mathrm{D}$, where the source release rate is low. This confirms that the source release rate parameter seems to have the highest impact on the performance when sensing with a MAV. In all cases, using a MCS lowers the distance overhead and decreases the variability of the results, highlighting the impact that a very accurate localization has on the performance. In this case, the average time taken to find the source was one minute and fifteen seconds. However, the results using an UWB localization system indicate that, despite being slower, the algorithm is effective in the four scenarios depicted and that this cheaper and easier to deploy system is a valuable alternative to the MCS.

Figure 5 shows the movement of the MAV as it was logged by the UWB system during a successful run with the sensor placed at the bottom. Two spiral movements can be seen, one closer to the source, marked with a red cross, and one at the beginning, as well as the initial Lévy Walk. Additionally, the figure shows that the drone is capable of following the plume for several meters and reacquire it in case of loss. Figure 6 shows the movement of the MAV logged by the MCS localization system during a successful run. It can be seen that the path is much smoother compared to the UWB one portrayed in Figure 5, especially corresponding to the spiral movements.

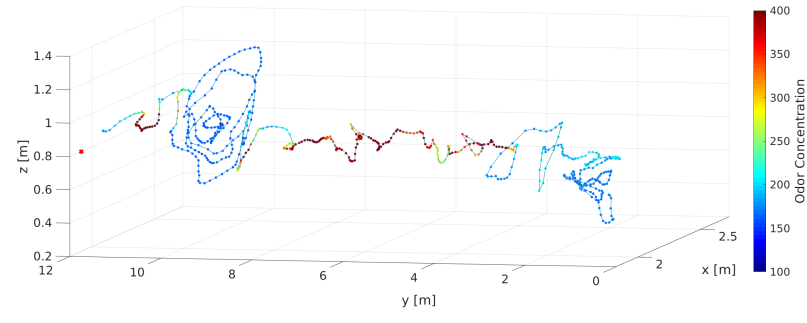

Fig. 5. Example of a successful run using the UWB localization system. The picture shows two spiral movements, one at the beginning and one at the end of the run, as well as the initial Lévy Walk.

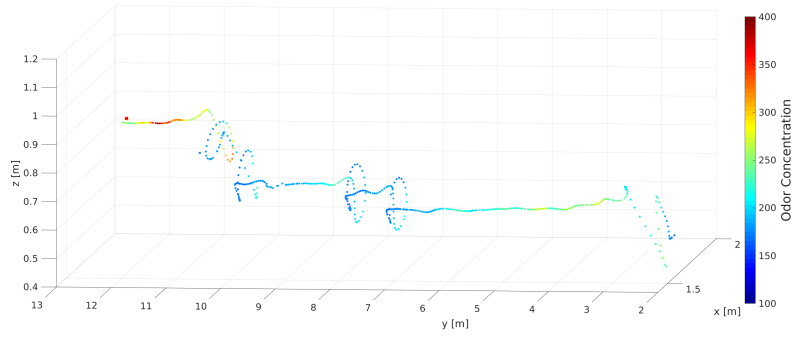

Fig. 6. Example of a successful run using the MCS localization system. The picture shows four spiral movements and a short Lévy Walk.

\section{CONCLUSIONS AND OUTLOOK}

The work presented in this paper focuses on the adaptation of a 3D bio-inspired algorithm for odor source localization for deployment on a MAV in a controlled physical environment represented by a wind tunnel, and the evaluation of its performance. The algorithm used in this paper was previously validated in the very same environment with a wheeled robot mounted on a traversing system (a 3-axis robotic manipulator present in many wind tunnels), but it did not take into account the peculiar challenges of implementing such an algorithm on a flying platform, which would be more suited for real-world deployment. Initially, an evaluation of the best sensor placement between top and bottom of the MAV's body is conducted. Experimental results show that the bottom sensor placement gives a higher success rate and a shorter distance overhead. After appropriate adaptations of the algorithm to be run on a MAV, a performance evaluation in different, repeatable environmental conditions is performed. It is shown that the adapted algorithm implemented on the MAV is robust to different environmental conditions, although a lower source release rate increases the distance overhead, and lower wind speeds affect the variability of the results. Finally, a comparison between an UWB localization system and a more accurate MCS-based system is carried out to quantify the impact of the localization accuracy on the algorithm performance. Results showed that a more accurate localization system has a significant impact on the performance of the algorithm. Thus, since the UWB approach offers a cheap and easy to deploy alternative to MCS and is still able to yield good results, its accuracy could be improved by integrating a time-of-flight laser sensor at the bottom of the drone as proposed in [5]. The next step in this 
work will also focus on improving the navigation strategy and embedding wind sensing capabilities in the MAV.

\section{REFERENCES}

[1] L. Marques, U. Nunes, and A. T. de Almeida, "Olfaction-based mobile robot navigation," Thin solid films, vol. 418, no. 1, pp. 51-58, 2002.

[2] M. Rossi, D. Brunelli, A. Adami, L. Lorenzelli, F. Menna, and F. Remondino, "Gas-drone: Portable gas sensing system on UAVs for gas leakage localization," in IEEE International Conference on Sensors, 2014, pp. 1431-1434.

[3] T. Kersnovski, F. Gonzalez, and K. Morton, "A UAV system for autonomous target detection and gas sensing," IEEE Aerospace Conference, pp. 1-12, 2017.

[4] M. Rossi and D. Brunelli, "Gas sensing on unmanned vehicles: Challenges and opportunities," in IEEE New Generation of Circuits and Systems Conference, 2017, pp. 117-120.

[5] S. Shigaki, M. Fikri, and D. Kurabayashi, "Design and experimental evaluation of an odor sensing method for a pocket-sized quadcopter," Sensors, vol. 18, no. 11, p. 3720, 2018.

[6] T. Villa, F. Salimi, K. Morton, L. Morawska, and F. Gonzalez, "Development and validation of a UAV based system for air pollution measurements," Sensors, vol. 16, no. 12, p. 2202, 2016.

[7] P. P. Neumann, H. Kohlhoff, D. Hüllmann, A. J. Lilienthal, and M. Kluge, "Bringing mobile robot olfaction to the next dimension-UAV-based remote sensing of gas clouds and source localization," in IEEE International Conference on Robotics and Automation, 2017, pp. 3910-3916.

[8] M. Rossi and D. Brunelli, "Autonomous gas detection and mapping with unmanned aerial vehicles," IEEE Transactions on Instrumentation and Measurement, vol. 65, no. 4, pp. 765-775, 2015.

[9] H. Ishida, "Blimp robot for three-dimensional gas distribution mapping in indoor environment," in AIP conference proceedings, vol. 1137, no. 1. AIP, 2009, pp. 61-64.

[10] J. Burgués, V. Hernández, A. J. Lilienthal, and S. Marco, "Smelling nano aerial vehicle for gas source localization and mapping," Sensors, vol. 19, no. 3, p. 478, 2019.

[11] P. P. Neumann, V. Hernandez Bennetts, A. J. Lilienthal, M. Bartholmai, and J. H. Schiller, "Gas source localization with a micro-drone using bio-inspired and particle filter-based algorithms," Advanced Robotics, vol. 27, no. 9, pp. 725-738, 2013.

[12] X. Chen and J. Huang, "Odor source localization algorithms on mobile robots: A review and future outlook," Robotics and Autonomous Systems, vol. 112, pp. 123-136, 2019.

[13] G. Kowadlo and R. A. Russell, "Robot odor localization: a taxonomy and survey," The International Journal of Robotics Research, vol. 27, no. 8, pp. 869-894, 2008.

[14] T. Lochmatter and A. Martinoli, "Tracking odor plumes in a laminar wind field with bio-inspired algorithms," in Experimental robotics. Springer, 2009, pp. 473-482.

[15] H. Ishida, K. Suetsugu, T. Nakamoto, and T. Moriizumi, "Study of autonomous mobile sensing system for localization of odor source using gas sensors and anemometric sensors," Sensors and Actuators A: Physical, vol. 45, no. 2, pp. 153-157, 1994.

[16] A. Lilienthal and T. Duckett, "Experimental analysis of gas-sensitive braitenberg vehicles," Advanced Robotics, vol. 18, no. 8, pp. 817-834, 2004.

[17] S. Shigaki, T. Sakurai, N. Ando, D. Kurabayashi, and R. Kanzaki, "Time-varying moth-inspired algorithm for chemical plume tracing in turbulent environment," IEEE Robotics and Automation Letters, vol. 3, no. 1, pp. 76-83, 2017.

[18] F. Rahbar, A. Marjovi, P. Kibleur, and A. Martinoli, "A 3-d bioinspired odor source localization and its validation in realistic environmental conditions," in IEEE/RSJ International Conference on Intelligent Robots and Systems, 2017, pp. 3983-3989.

[19] G. Ferri, E. Caselli, V. Mattoli, A. Mondini, B. Mazzolai, and P. Dario, "Spiral: A novel biologically-inspired algorithm for gas/odor source localization in an indoor environment with no strong airflow," Robotics and Autonomous Systems, vol. 57, no. 4, pp. 393-402, 2009.

[20] SGX Sensortech technologies, "MiCS-5521 CO/VOC sensor," http://www.sgxsensortech.com.

[21] T. Lochmatter and A. Martinoli, "Theoretical analysis of three bioinspired plume tracking algorithms," in IEEE International Conference on Robotics and Automation, 2009, pp. 2661-2668. 